\title{
Improvement in Loss to Follow-up of Newborn Hearing Screening: A lesson from Louisiana Early Hearing Detection and Intervention Program
}

\author{
Xiaoling Ye*1, Tri Trann, 3, Mary Jo Smith², Jeanette Webb², Terri Mohren² and Melinda \\ Peat $^{2}$ \\ ${ }^{1}$ Tulane School of Public Health and Tropical Medicine, Department of Epidemiology, new orleans, LA, USA; 'Louisiana DHH OPH \\ CSHS Early Hearing Detection and Intervention Program, new orleans, LA, USA; ${ }^{3}$ LSUHSC School of Medicine, Department of \\ Pediatrics, new orleans, LA, USA
}

\section{Objective}

The study aimed to analyze the trend in loss to follow-up of hearing screening among newborns who failed initial hearing screening tests prior to hospital discharge, and evaluate accomplishments in improving loss to follow-up from 2004 to 2011 in Louisiana.

\section{Introduction}

Newborns who fail initial hearing screening are encouraged to follow up to get further tests to confirm diagnosis of hearing loss. Loss to follow-up of hearing screening is defined as a newborn who fails the initial hearing test before hospital discharge but does not follow up with further hearing tests to confirm diagnosis of hearing loss. Although rates of loss to follow-up are considered high in Louisiana, with great efforts by Louisiana Early Hearing Detection and Intervention Program (EHDI), loss to follow-up has significantly improved in the last several years. A trend analysis of loss to follow-up was helpful in evaluating accomplishments to reduce loss to follow-up and refining plans and strategies to enhance the quality of newborn hearing detection and intervention in the state.

\section{Methods}

The data of Louisiana Early Hearing Detection and Intervention (EHDI) Surveillance linked with birth certificates were used for analysis. The study included 24,516 newborns who were born between 2007 and 2011 in Louisiana and failed initial hearing screening tests prior to hospital discharge. Both loss to follow-up of hearing screening which were and were not confirmed by audiologists were combined as total loss to follow-up. Unadjusted and adjusted log-linear models were conducted to estimate crude and adjusted annual percent change of rates (\%) in loss to follow-up. Variables defining characteristics of newborns and mothers derived from birth certificates were controlled for in adjusted models. In addition to trends analyzed from 2004 to 2011 in all races, trends were analyzed by race/ethnicity and time periods before and after 2007 when a major reduction of the rate of loss to follow-up occurred through significant efforts by the EHDI Program. Linkpro 3.0 and SAS 9.3 were used for data linkage and analyses. Alpha was set at .05 for statistical significance.

\section{Results}

Overall, the rate of loss to follow-up was around 50\% from 2004 to 2006. It dropped to about 35\% in 2007 and kept stable until 2010, and then dropped to $27 \%$ in 2011. In adjusted models, the annual percent change (APC) of loss to follow-up rates was $-8.56,-3.33$, and -7.58 between 2004-2011, 2004-2006, and 2008-2011, respectively for all races. When stratified by race/ethnicity, the APC was $-9.55,-7.12$, and -6.60 between 2004-2011, 2004-2006, and 2008-2011, respectively, for Non-Hispanic white; $-7.71,-0.52$, and -7.06 between 20042011, 2004-2006, and 2008-2011, respectively, for Non-Hispanic black; and -8.96, -0.24, and -13.20 between 2004-2011, 2004-2006, and 2008-2011, respectively, for Hispanic. Trends were significantly reduced during all periods and in groups of race/ethnicity except for Non-Hispanic black and Hispanic between 2004 and 2006 (p values $>.05$ ). APCs were significantly higher in the period 2004-2006 than in period 2008-2011. A major drop in loss to follow-up rate occurring in 2007 with the APC of $31 \%$ was considered as a result of successful interventions, which included hiring follow-up coordinators, holding individual hospital meetings with birthing hospitals, frequently organizing regional LA EHDI Task Force meetings, and providing follow-up reports to LA EHDI hospital supervisors.

\section{Conclusions}

A significant improvement in loss to follow-up after failure to pass newborn hearing screening was contributed to effective cooperation between LA EHDI Program and stakeholders as well as to outreach activities and patient contact, which are strongly encouraged to continue and expand.

\section{Keywords}

Trend analysis; Loss to follow-up; Newborn hearing screening; Data linkage

*Xiaoling Ye

E-mail: janice.ye268@gmail.com 